\title{
Ultralow-Power Electronic Trapping of Nanoparticles with Sub-10 nm Gold Nanogap Electrodes
}

\author{
Avijit Barik ${ }^{1,2}$, Xiaoshu Chen ${ }^{l}$, and Sang-Hyun $\mathrm{Oh}^{1,2^{*}}$ \\ ${ }^{1}$ Department of Electrical and Computer Engineering, \\ ${ }^{2}$ Department of Biomedical Engineering, \\ University of Minnesota, Minneapolis, MN 55455, U.S.A. \\ *E-mail: sang@umn.edu
}




\section{Methods}

Atomic layer lithography. An array of $8 \mathrm{~mm} \times 1 \mathrm{~mm}$ rectangular patterns was created on a glass wafer by standard photolithography using a negative photoresist NR71-1500P. Next, 150 $\mathrm{nm}$ gold (with $3 \mathrm{~nm}$ chromium as an adhesion layer) was deposited using an electron-beam evaporator (Temescal), followed by a lift-off step (Microposit remover 1165) to create rectangular openings in the gold film. Subsequently, $9 \mathrm{~nm}$ thick $\mathrm{Al}_{2} \mathrm{O}_{3}$ layer was deposited by atomic layer deposition (ALD, Cambridge NanoTech Inc., Savannah) at $250{ }^{\circ} \mathrm{C}$, which coats the surface conformally at the deposition rate of $\sim 1 \AA$ cycle. The thickness of the $\mathrm{Al}_{2} \mathrm{O}_{3}$ layer was calibrated using ellipsometry (Gaertner). Next, $141 \mathrm{~nm}$ of gold (without adhesion) was deposited by electron-beam evaporation (Temescal). The thickness of this second metal layer was chosen so that there was no contact with the first metal layer. The metal deposited outside the trench was peeled off using single-sided tape (3M Scotch Magic ${ }^{\mathrm{TM}}$ Tape) without leaving any residue.

Electrode fabrication. To make electrical connections with the metal inside the rectangular pattern, a $72 \mathrm{~nm}$ gold film with a $3 \mathrm{~nm}$ titanium adhesion layer was sputtered on one of the longer sides of the rectangle using an AJA sputterer, while the other side was protected. Next, an electrode design was created using photolithography (negative photoresist, NR71-1500P), containing the nanogaps of desirable length in the center with contact pads for electrical connection on either side. The residual metal, which was not protected by photoresist, was etched away by bombarding the device with argon ions at an angle of $75^{\circ}$ in an ion mill (Intlvac Nanoquest). This step created electrically isolated electrodes separated by $\mathrm{Al}_{2} \mathrm{O}_{3}$-filled nanogap. Finally, the photoresist was removed and the gaps were tested for any unwanted electrical connections using a current voltage (IV) measurement setup (HP 4145A). Only electrically isolated gaps were used for DEP experiments. 
DEP experiments using nanogap electrodes. A solution volume of $\sim 4 \mu 1$ was placed on top of the devices, confined within a PDMS ring. The solution was covered by a cover slip on top to avoid any unwanted evaporation. An AC bias was applied across the nanogaps using a function generator (HP 33120A). The peak amplitude of the voltage used in most experiments was less than or equal to $1 \mathrm{~V}$. Depending on the particle, appropriate frequencies were chosen for positive and negative DEP based on their characteristic CM factor plot. Most of the solutions were prepared in water of conductivity $\sim 4 \mu \mathrm{S} / \mathrm{cm}$ (measured by B-771 LAQUAtwin, Horiba Scientific) and for experiments involving buffers, solutions were prepared in $1 \%$ phosphate buffered saline (PBS) of conductivity $\sim 180 \mu \mathrm{S} / \mathrm{cm}$ or $10 \%$ PBS of conductivity $\sim 1.71 \mathrm{mS} / \mathrm{cm}$.

Fluorescence microscopy. Various fluorescent nanoparticles that were used in this work include $190 \mathrm{~nm}$ diameter carboxylate-modified polystyrene beads $\left(\lambda_{e x}: 470 \mathrm{~nm}, \lambda_{e m}: 525 \mathrm{~nm}\right.$, Bangs Laboratories); $30 \mathrm{~nm}$ diameter carboxylate-modified polystyrene beads $\left(\lambda_{\text {ex }}: 470 \mathrm{~nm}, \lambda_{\text {em }}: 505\right.$ nm, Sigma-Aldrich); high-pressure, high-temperature monocrystalline and carboxylate-modified nanodiamonds of type $\mathrm{lb}$ containing 10-15 NV centers, with average volumetric size of $40 \mathrm{~nm}$ $\left(\lambda_{e m}: 637 \mathrm{~nm}\right.$, Adamas Nanotechnologies); and carboxylate-modified CdSe quantum dots of core/shell size $8 \mathrm{~nm}\left(\lambda_{e m}: 625 \mathrm{~nm}\right.$, Qdot 625 ITK Carboxyl Quantum Dots, Life Technologies). A laser driven light source (Energetiq) was passed through the appropriate filters to excite fluorescence and the emitted light was collected through another filter. A $50 \times$ objective was used to observe diffraction-limited individual nanoparticles while using an image acquisition time of $200 \mathrm{~ms}$ for $190 \mathrm{~nm}$ beads, $500 \mathrm{~ms}$ for $30 \mathrm{~nm}$ beads and quantum dots, and $1 \mathrm{~s}$ for nanodiamond particles. Fluorescence images were collected at regular intervals using a Photometrics CoolSNAP HQ2 CCD camera and Micro-Manager software. Fluorescent images were further analyzed using ImageJ software. 
FEM simulation. The static electric field around the nanogap electrodes was simulated with a two-dimensional FEM model using COMSOL Multiphysics package. The nanogaps were modeled as two gold electrodes of thickness $150 \mathrm{~nm}$, separated by a dielectric $\left(\mathrm{Al}_{2} \mathrm{O}_{3}\right)$ layer of specific thickness. For the electric field map comparison between microelectrodes and nanogap electrodes (Fig. 2a), a voltage of $1 \mathrm{~V}$ was applied across a gap width of $1 \mu \mathrm{m}$ and $10 \mathrm{~nm}$, respectively. The minimum grid size parallel to the gold surface and within the gap was $\sim 0.2 \mathrm{~nm}$ and a set of 8 boundary layers were added to all the boundaries where a strong electric field gradient was present. A data collection point to calculate the DEP force (proportional to $\nabla|E|^{2}$ ) was chosen at various distances away from the center of the nanogap. The minimum trapping voltage was calculated by comparing the DEP force with the thermal force for different sizes of particles. 

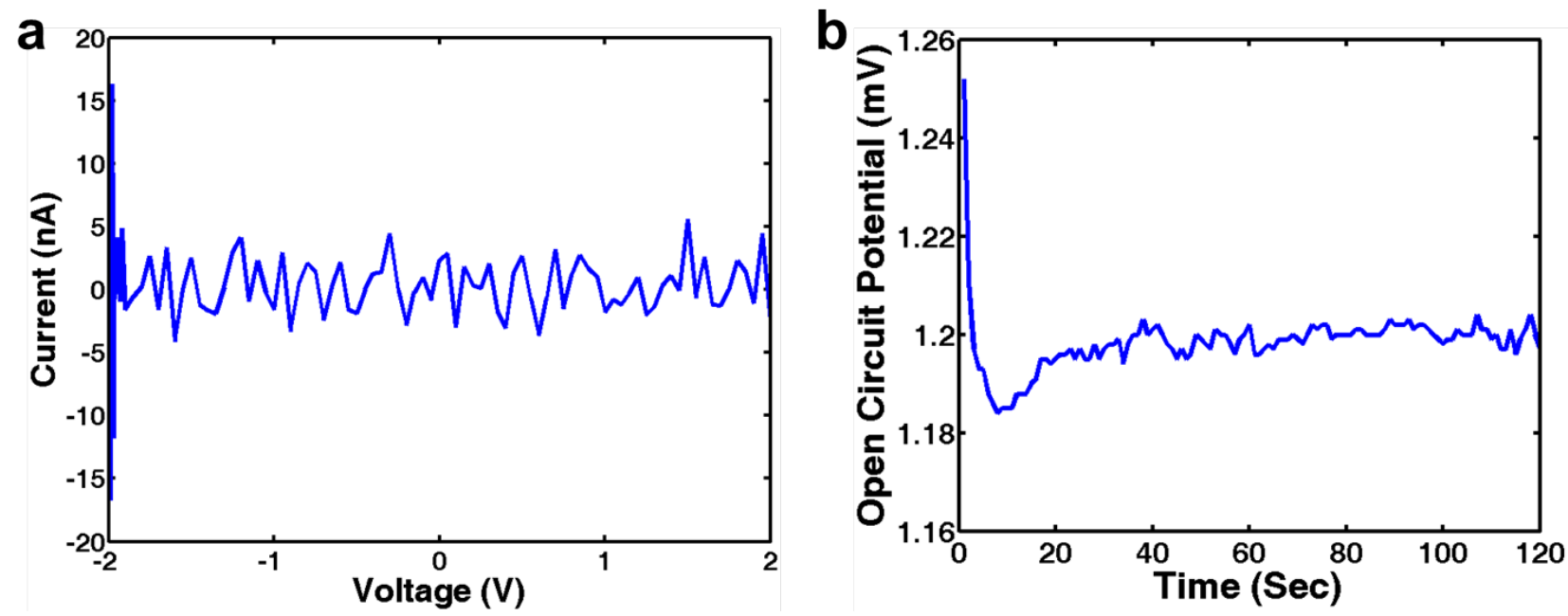

Figure S1. (a) Sample current-voltage (IV) curve obtained from $9 \mathrm{~nm}$ gap devices. It shows the characteristic noise of the IV workstation while scanning the voltage from -2 to $+2 \mathrm{~V}$ at a step size of $0.05 \mathrm{~V}$. (b) Open circuit potential of the nanogap device was measured using a electrochemical workstation (CH Instruments). Approximately $1.2 \mathrm{mV}$ was observed, which is significantly lower than the working voltage range used during the experiments. 


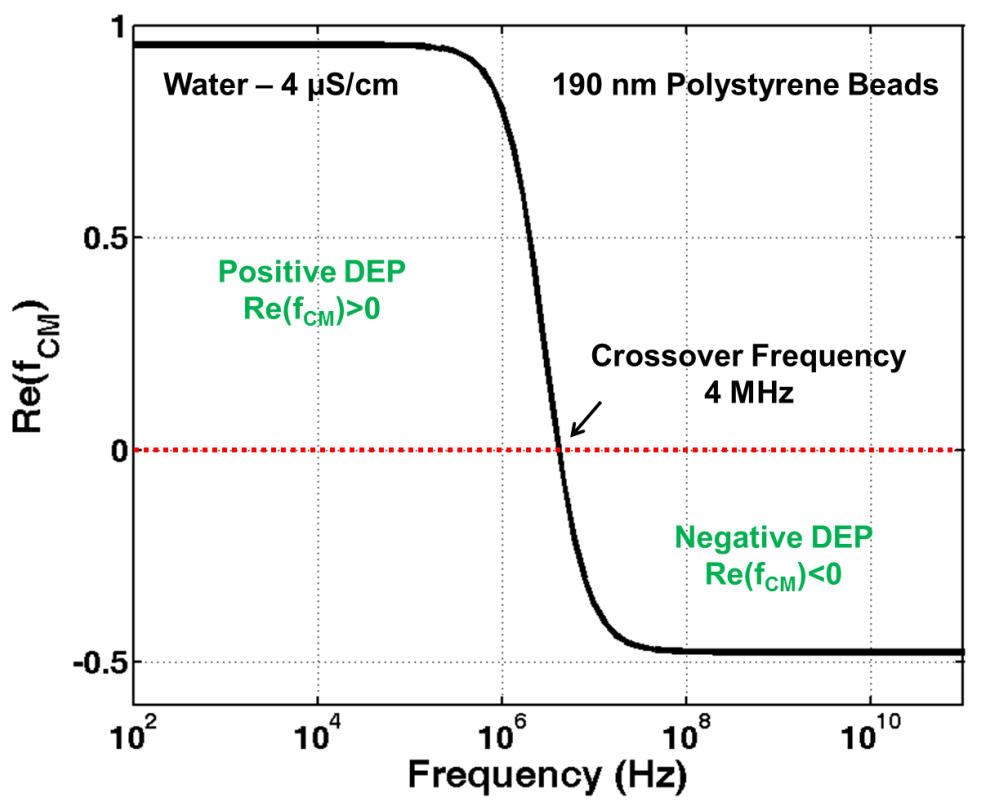

Figure S2. Clausius-Mossotti (CM) factor plot for $190 \mathrm{~nm}$ polystyrene beads in water (conductivity: $4 \mu \mathrm{S} / \mathrm{cm}$ ). Particles are attracted toward the gap by positive DEP and repelled away from the gap by negative DEP. 


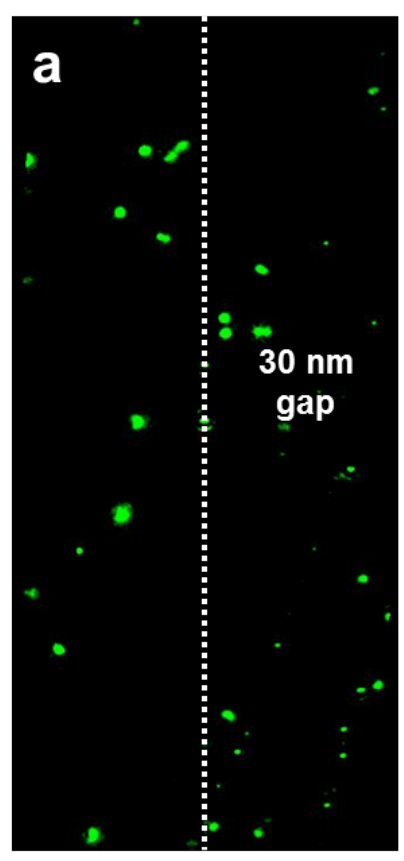

Before DEP

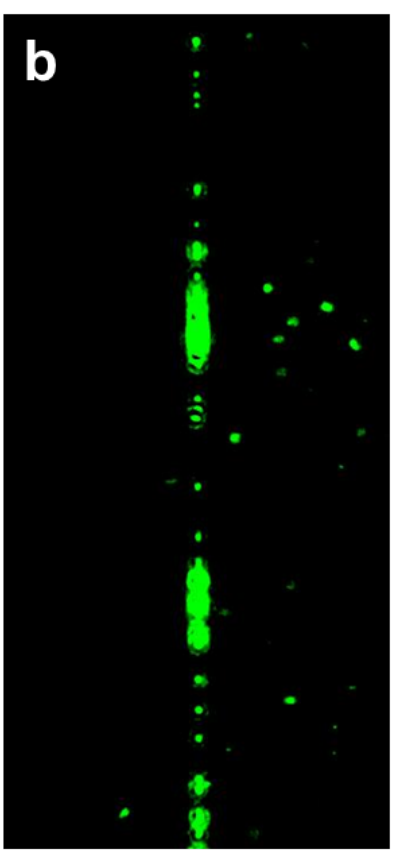

Positive DEP

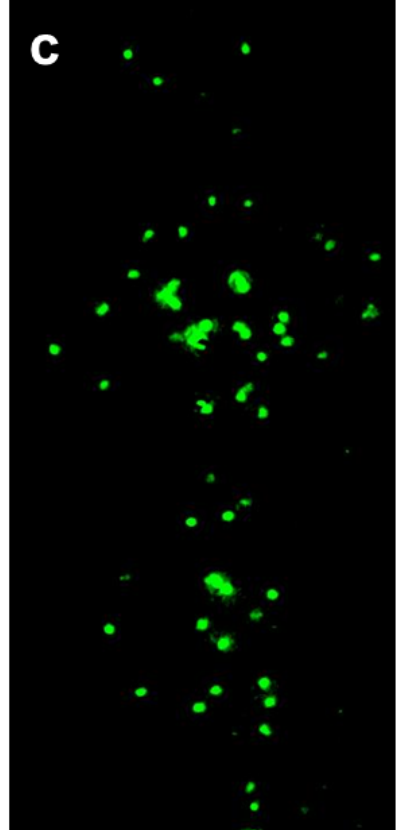

Negative DEP

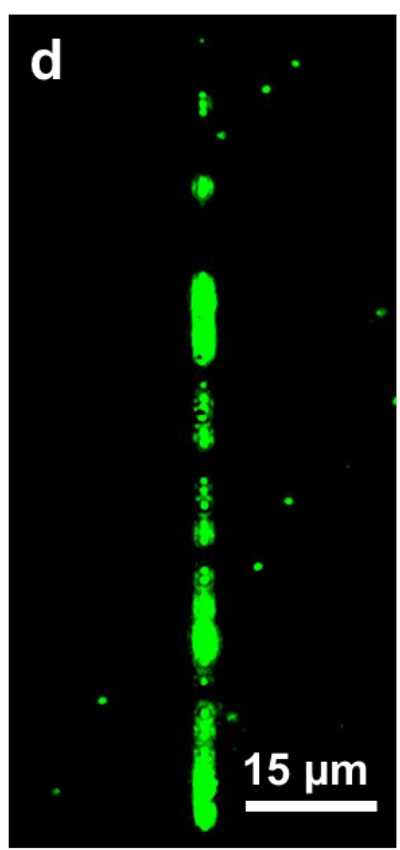

Positive DEP

Figure S3. Trapping of $190 \mathrm{~nm}$ polystyrene beads on a 30-nm-gap device. (a) Fluorescent image of floating beads before turning on the bias. (b) Beads are trapped along the nanogap by positive DEP when a bias of peak amplitude $1 \mathrm{~V}$ is applied at a frequency of $1 \mathrm{MHz}$. (c) The beads can be released from the gap by negative DEP at a frequency of $10 \mathrm{MHz}$. (d) Trapping is reversible. False-color fluorescent images are used to represent the emission wavelength. 


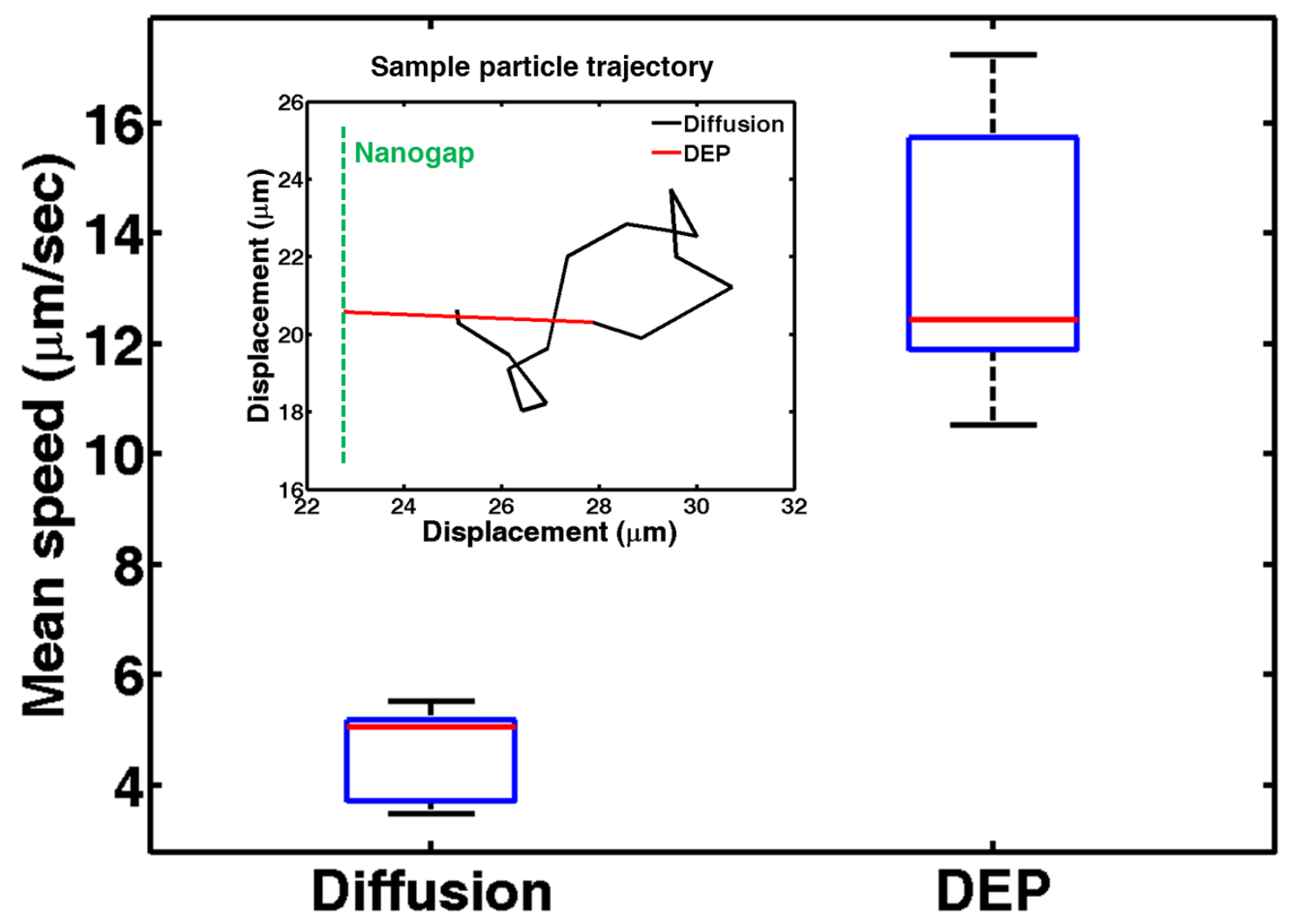

Figure S4. A two-dimensional particle tracking software was used to track the trajectories of five different particles during diffusion and under the influence of the DEP force. An example trajectory is shown in the inset, where the movement due to DEP is noted in red. Furthermore the average speed of the particles was calculated during random motion due to diffusion and for deterministic movement due to DEP. For the bead diameter of $190 \mathrm{~nm}$ and an applied voltage of $1 \mathrm{~V}$, a bead can be trapped from 5-10 $\mu \mathrm{m}$ away from the gap, which further demonstrates the strength of our device. 


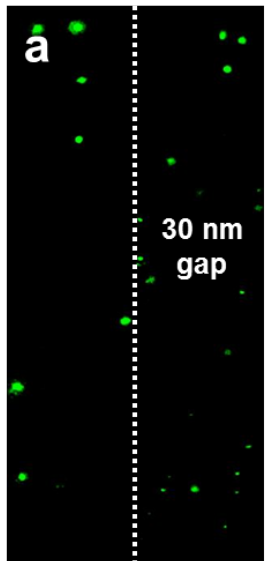

Before DEP

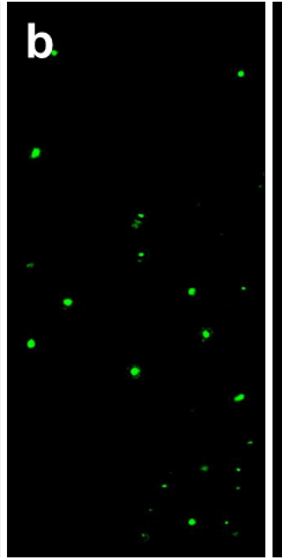

$400 \mathrm{mV}$

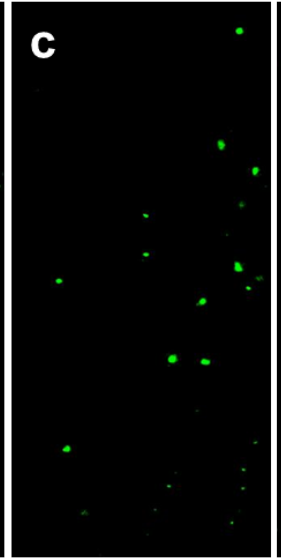

$500 \mathrm{mV}$

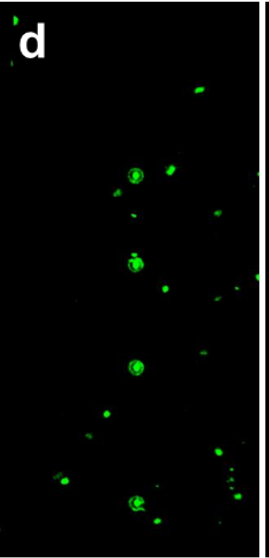

$600 \mathrm{mV}$

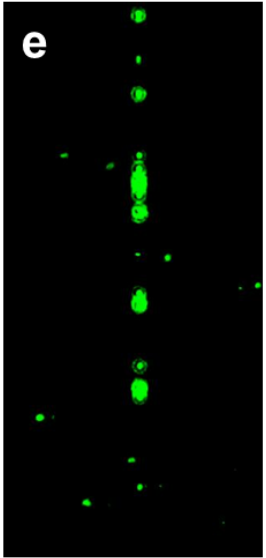

$700 \mathrm{mV}$

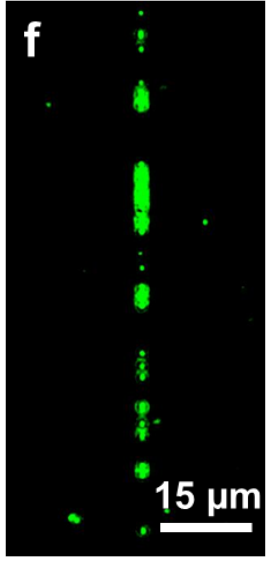

$800 \mathrm{mV}$

Figure S5. Time-lapse fluorescent images represent the voltage dependence study of the $190 \mathrm{~nm}$ polystyrene beads on a 30-nm-gap electrode. The minimum trapping voltage was $600 \mathrm{mV}-$ increasing the voltage further enables trapping of even more number of beads. Reducing the width of the nanogap to sub-10 nm helps to trap these particles at an even lower voltage of 300 $\mathrm{mV}$ as shown in Figures $2 \mathrm{c}-\mathrm{f}$ in the main text. This further corroborates the dependence of gap width on the trapping efficiency of the device. False-color fluorescent images are used to represent the emission wavelength. 

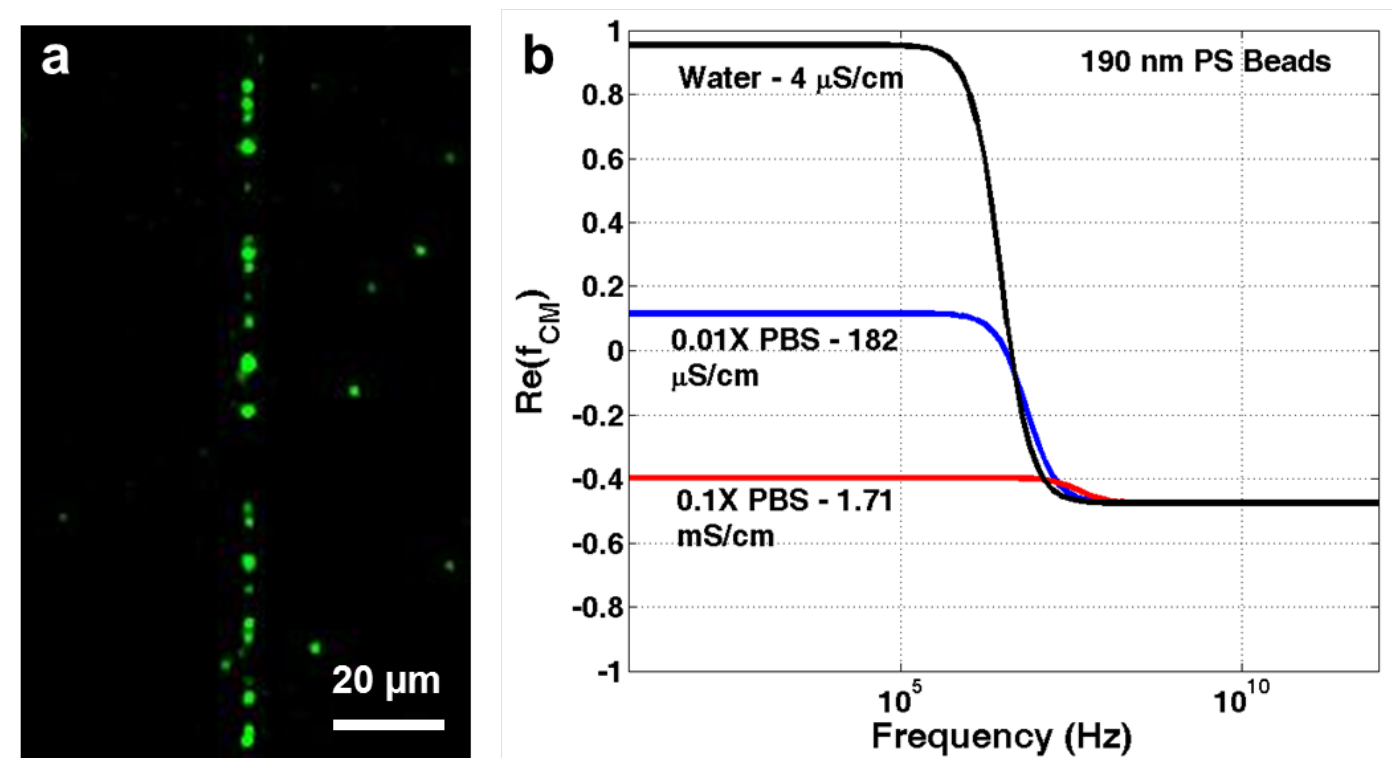

Figure S6. Trapping of $190 \mathrm{~nm}$ polystyrene beads in 0.01X PBS. (a) Shows trapping of $190 \mathrm{~nm}$ polystyrene beads on a 30-nm-gap device in 0.01X PBS while applying $1 \mathrm{~V}$ at $1 \mathrm{MHz}$ frequency. Increasing the conductivity further shifts the $\mathrm{CM}$ factor completely into the negative regime hindering trapping. (b) $\mathrm{CM}$ factor plot shows the dependence of trapping behavior on the conductivity of the surrounding medium. As the medium conductivity is increased, it becomes more challenging to trap the beads on the gap. False-color fluorescent image is used to represent the emission wavelength. 